\title{
Imaging features of pancreatic metastases: A comparison with pancreatic ductal adenocarcinoma
}

\author{
Massimo Galia $^{\mathrm{a}}$, Domenico Albano ${ }^{\mathrm{a}, *}$, Dario Picone ${ }^{\mathrm{a}}$, Maria Chiara Terranova ${ }^{\mathrm{a}}$, Antonino Agrusa ${ }^{\mathrm{b}}$, \\ Giuseppe Di Buono ${ }^{b}$, Annalisa Licata ${ }^{c}$, Giuseppe Lo Re ${ }^{a}$, Ludovico La Grutta ${ }^{a}$, Massimo Midiri ${ }^{a}$ \\ a Department of Radiology, Di.Bi.Med., University of Palermo, Via del Vespro 127, 90127 Palermo, Italy \\ ${ }^{\mathrm{b}}$ Department of General Surgery, Urgency and Organ Transplantation, University of Palermo, Via del Vespro 127, 90127 Palermo, Italy \\ c Gastroenterology and Hepatology Section, Di.Bi.Mis., University of Palermo, Via del Vespro 127, 90127 Palermo, Italy
}

\section{A R T I C L E I N F O}

\section{Keywords:}

Computed tomography

Magnetic resonance

Abdominal radiology

Pancreas

Metastases

Adenocarcinoma

\begin{abstract}
A B S T R A C T
Purpose: To compare imaging features of pancreatic metastases (PM) with those of pancreatic ductal adenocarcinomas (PDAC).

Methods: CT and MR scans of 24 patients with 54 PM and 30 patients with PDAC were reviewed to evaluate the imaging features, which were compared by using a Chi square test.

Results: We found a statistically significant difference between PM and PDAC based on location $(P<0.001)$, margins $(P<0.001)$, arterial enhancement $(P=0.004)$, rim enhancement $(P<0.001)$, pancreatic duct dilatation $(P=0.01)$, common bile duct dilatation $(P=0.003)$, vascular involvement $(P=0.02)$, parenchymal atrophy $(P<0.001)$, peripancreatic fluid $(P=0.03)$.

Conclusion: Imaging features might be helpful to differentiate PM from PDAC.
\end{abstract}

\section{Introduction}

Pancreatic malignancies are mainly primary exocrine pancreatic neoplasms, whereas neuroendocrine tumors are much less common [1]. Secondary pancreatic neoplasms are rare, accounting from $2 \%$ to $5 \%$ of all malignant lesions of the pancreas [2]. As demonstrated by autopsy series, one third of pancreatic metastases (PM) are clinically misdiagnosed as primary malignancies [3]. Indeed, these lesions have no specific symptoms and usually occur in advanced stage neoplasms, when the clinical picture is already severe. Nevertheless, the pancreas may be the only secondary site of a neoplasm, especially in renal cell carcinoma [4], and the early diagnosis of PM may change the treatment and prognosis of the disease. In the differential diagnosis of pancreatic lesions, cancer antigens have limited diagnostic reliability [5].

Imaging modalities such as Computed Tomography (CT) and Magnetic Resonance (MR) are routinely performed during the follow-up of oncologic patients [6-10]. In this setting CT and MR may play a crucial role in the early identification of PM, but their part in the management of PM has been evaluated only on small series of patients [11-19].

Thus, the aims of our study were: (i) to review the CT and MR scans performed on patients with PM at our Institution to describe the imaging features of these lesions; (ii) to compare imaging features of PM with those of pancreatic ductal adenocarcinomas (PDAC).

\section{Materials and methods}

\subsection{Patients}

We performed a search of our Institution database to identify all cases of PM found on CT and MR scans from 2006 to 2016. The inclusion criterion was the presence of PM confirmed by histology or by follow-up examinations on patients receiving chemotherapy treatments. We excluded those cases with direct pancreatic invasion by a neoplasm from an adjacent organ. On the basis of this selection, our study population consisted of 24 patients (12 males, 12 females; mean age: 61, range 52-83). Six patients had two CT examinations, 12 patients had three CT examinations, 6 patients had 4 CT examinations. In addition to CT, 13 patients had MR examinations ( 8 patients had one MR and 5 patients had 2 MRs). Therefore we reviewed 90 examinations (72 CT and $18 \mathrm{MR}$ ). Follow-up CT imaging was available in all patients (mean 25 months, range 4-33 months) while follow-up MR imaging was available in five patients (mean 8 months, range $3-17$ months). Moreover, we reviewed the CT and MR examinations performed on 30 patients (12 males, 18 females; mean age: 73, range 47-95) with histologically confirmed PDAC.

This retrospective study was approved by the Institutional Review Board with a waiver of the requirement for informed consent.

\footnotetext{
* Corresponding author.

E-mail address: albanodomenico@me.com (D. Albano).
} 
Table 1

Distribution of primary malignancies and extra-pancreatic metastases of 24 patients with pancreatic metastases.

\begin{tabular}{ll}
\hline Site & Number (\%) \\
\hline Primary malignancy & \\
Lung carcinoma & $8 / 24(33 \%)$ \\
Renal cell carcinoma & $6 / 24(25 \%)$ \\
Thyroid cancer & $4 / 24(17 \%)$ \\
Breast carcinoma & $2 / 24(8 \%)$ \\
Merkeloma cancer & $2 / 24(8 \%)$ \\
Adrenal gland cancer & $1 / 24(4 \%)$ \\
Soft tissue liposarcoma & $1 / 24(4 \%)$ \\
Extra-pancreatic metastases & \\
Lung & $18 / 24(75 \%)$ \\
Lymph nodes & $14 / 24(58 \%)$ \\
Liver & $12 / 24(50 \%)$ \\
Adrenal gland & $10 / 24(42 \%)$ \\
Kidney & $6 / 24(25 \%)$ \\
Muscles & $6 / 24(25 \%)$ \\
Peritoneum & $6 / 24(25 \%)$ \\
Bone & $4 / 24(17 \%)$ \\
Pleura & $2 / 24(8 \%)$ \\
Pericardium & $2 / 24(8 \%)$ \\
Brain & $2 / 24(8 \%)$ \\
Skin & $2 / 24(8 \%)$ \\
Bowel & $2 / 24(8 \%)$ \\
\hline
\end{tabular}

Table 2

Imaging features of 54 pancreatic metastases and 30 primary pancreatic adenocarcinomas.

\begin{tabular}{|c|c|c|c|}
\hline Imaging characteristics & Metastases & Adenocarcinoma & $P$-value \\
\hline Size (range) & $2.5 \mathrm{~cm}(1.2-4.3)$ & $4.7 \mathrm{~cm}(1-9.4)$ & \\
\hline Location & & & $<0.001$ \\
\hline Head & $6 / 54(11 \%)$ & $17 / 30(57 \%)$ & \\
\hline Neck & $6 / 54(11 \%)$ & $2 / 30(7 \%)$ & \\
\hline Body & $20 / 54(37 \%)$ & $6 / 30(20 \%)$ & \\
\hline Tail & $22 / 54(41 \%)$ & $5 / 30(17 \%)$ & \\
\hline Margins & & & $<0.001$ \\
\hline Well-defined & $21 / 54(39 \%)$ & $0 / 30(0 \%)$ & \\
\hline Ill-defined & $19 / 54(35 \%)$ & $23 / 30(77 \%)$ & \\
\hline Lobulated & $14 / 54(26 \%)$ & $7 / 30(23 \%)$ & \\
\hline Attenuation on unenhanced CT & & & 0.344 \\
\hline Hypodense & $23 / 54(43 \%)$ & $16 / 30(53 \%)$ & \\
\hline Isodense & $31 / 54(57 \%)$ & $14 / 30(47 \%)$ & \\
\hline Hyperdense & $0 / 54(0 \%)$ & $0 / 30(0 \%)$ & \\
\hline Enhancement on arterial phase & & & 0.004 \\
\hline Hypovascular & $38 / 54(70 \%)$ & $29 / 30(97 \%)$ & \\
\hline Hypervascular & $16 / 54(30 \%)$ & $1 / 30(3 \%)$ & \\
\hline Enhancement on venous phase & & & 0.057 \\
\hline Homogeneous & $15 / 54(28 \%)$ & $3 / 30(10 \%)$ & \\
\hline Heterogeneous & $39 / 54(72 \%)$ & $27 / 30(90 \%)$ & \\
\hline Rim enhancement & $22 / 54(41 \%)$ & $1 / 30(3 \%)$ & $<0.001$ \\
\hline Calcifications & $2 / 54(4 \%)$ & $3 / 30(10 \%)$ & 0.835 \\
\hline Main pancreatic duct dilatation & $6 / 54(11 \%)$ & $18 / 30(60 \%)$ & 0.01 \\
\hline Common bile duct dilatation & $3 / 54(6 \%)$ & $15 / 30(50 \%)$ & 0.003 \\
\hline Vascular involvement & $7 / 54(13 \%)$ & $18 / 30(60 \%)$ & 0.02 \\
\hline Parenchymal atrophy & $3 / 54(6 \%)$ & $17 / 30(57 \%)$ & $<0.001$ \\
\hline Peripancreatic fluid & $4 / 54(7 \%)$ & $13 / 30(43 \%)$ & 0.003 \\
\hline Pancreatitis & $0 / 54(0 \%)$ & $2 / 30(7 \%)$ & 0.197 \\
\hline
\end{tabular}

\subsection{CT protocol}

Patients underwent CT scan with a 64-slice CT scanner (Brilliance 64, Philips Medical System, Cleveland, Ohio, USA) and 128-slice CT scanner (Definition AS +, Siemens Healthcare, Forchheim, Germany). All patients drank $500-800 \mathrm{ml}$ of water immediately before undergoing imaging to distend the stomach and duodenum. Unenhanced images of the pancreas initially were obtained by using $3 \mathrm{~mm}$ collimation to define the cranio-caudal extent of the pancreas. Then, by using dual head-power automatic injector (Stellant, MedRAD, Pittsburgh, PA, USA) connected to an 18-gauge needle cannula placed in an antecubital vein, a bolus of $100-120 \mathrm{ml}$ of non-ionic iodinated contrast agent (Iomeprol, Iomeron 400, Bracco, Milan, Italy) followed by a saline flushing of $20-30 \mathrm{ml}$ was administered at an injection rate of 3-4 ml/s. For dynamic phase imaging, pancreatic parenchymal, portal and late phases, were performed following a scanning delay of $23 \mathrm{~s}, 57 \mathrm{~s}$ and $163 \mathrm{~s}$, respectively, after the attenuation of a region-of-interest positioned in the aorta at the level of the celiac trunk reached $100 \mathrm{HU}$. The acquisition parameters were: tube voltage, $120 \mathrm{kV}$; collimation, 64/ $128 \times 0.6 \mathrm{~mm}$; rotation time, $0.5 \mathrm{~s}$; pitch, 0.6 .

\subsection{MR protocol}

All patients were imaged with a 1.5T-MR imaging unit (Signa Excite, General Electric, Health care, Milwaukee, WI, USA). A dedicated abdominal multichannel surface coil was used for all patients. Imaging protocol included axial pre-contrast images acquired with T2-weighted fast-spin echo sequence (TR/TE, $4000 / 76 \mathrm{~ms}$; section thickness $5-6 \mathrm{~mm})$ and T1-weighted axial in-phase and out-of-phase gradientrecalled-echo (GRE) sequence (TR/TE, 140/2.2-4.4 ms; section thickness, $5-6 \mathrm{~mm}$ ). Those examinations performed for the evaluation of pancreatic lesions included two-dimensional and three-dimensional MR cholangiography sequences. Dynamic studies were performed with three-dimensional fat-suppressed T1-weighted GRE sequence (LAVATR/TE, 3.8/1.2 ms; FA 12; slice thickness: $4.4 \mathrm{~mm}$; intersection gap $2 \mathrm{~mm}$; FOV: $44 \mathrm{~cm}$; matrix $256 \times 256$ ) using a bolus-tracking system. Images were acquired in the axial plane immediately before and after intravenous injection of either $0.1 \mathrm{mmol} / \mathrm{kg}$ body weight of gadobenate dimeglumine at $2 \mathrm{ml} / \mathrm{s}$ or $0.025 \mathrm{mmol} / / \mathrm{kg}$ body weight of gadoxetic acid at $1 \mathrm{ml} / \mathrm{s}$ through a 20-gage intravenous catheter by means of a power injector (Medrad Spectris Solaris EP MR Injection System; Bayer Healthcare), followed by a $20-\mathrm{ml}$ saline flush at the same injection rate. Scanning delays after automatic detection of contrast bolus were 18,60 , $180 \mathrm{~s}$ and $300 \mathrm{~s}$, respectively, for the acquisition of the arterial, portal venous, 3-min, and 5-min phase. The choice of contrast agent was based on availability and personal preferences of the radiologist. Finally, all patients underwent diffusion-weighted imaging sequence as follow: single-shot spin-echo echo-planar with chemical-shift selective fatsuppression technique; scan direction, axial; respiration, non-breathhold method; b value, $0 \mathrm{~s} / \mathrm{mm}^{2}, 150 \mathrm{~s} / \mathrm{mm}^{2}$ and $600 \mathrm{~s} / \mathrm{mm}^{2}$ (with diffusion weighted gradients applied in three orthogonal directions); TR/ TE, 8000/73 ms; inversion time, $70 \mathrm{~ms}$; matrix, $128 \times 64$; slice thickness/gap, $5 \mathrm{~mm} / 0 \mathrm{~mm}$; field of view, $40 \mathrm{~cm}$; number of excitations, 6 ; and acquisition time, approximately $5 \mathrm{~min}$.

\subsection{Image interpretation and statistical analysis}

CT and MR images were independently reviewed by two radiologists with 16 and 9 years of experience in oncology imaging, respectively. Disagreements were resolved by consensus. Since the histologic confirmation of PM was available in few cases, the morphological and size changes of these lesions detected during treatment was used as proof of their nature. In patients with PM, we assessed the location of primary malignancies and the presence of extra-pancreatic metastases. Then, in both patients with PM and those with PDAC, the following imaging features were evaluated: site, number, size, margins, density/signal intensity of lesions and healthy parenchyma on non-contrast and contrast enhanced images, signal intensity on high b-value DWI images, enhancement pattern on arterial (hypovascular/hypervascular) and venous phase (homogeneous/heterogeneous), rim enhancement, calcifications, main pancreatic duct dilatation ( $>2.5 \mathrm{~mm}$ ), common bile duct dilatation $(>7 \mathrm{~mm}$ ), vascular involvement, atrophic parenchyma, peripancreatic fluid, pancreatitis. The Chi square test was used to compare the imaging features of PM 


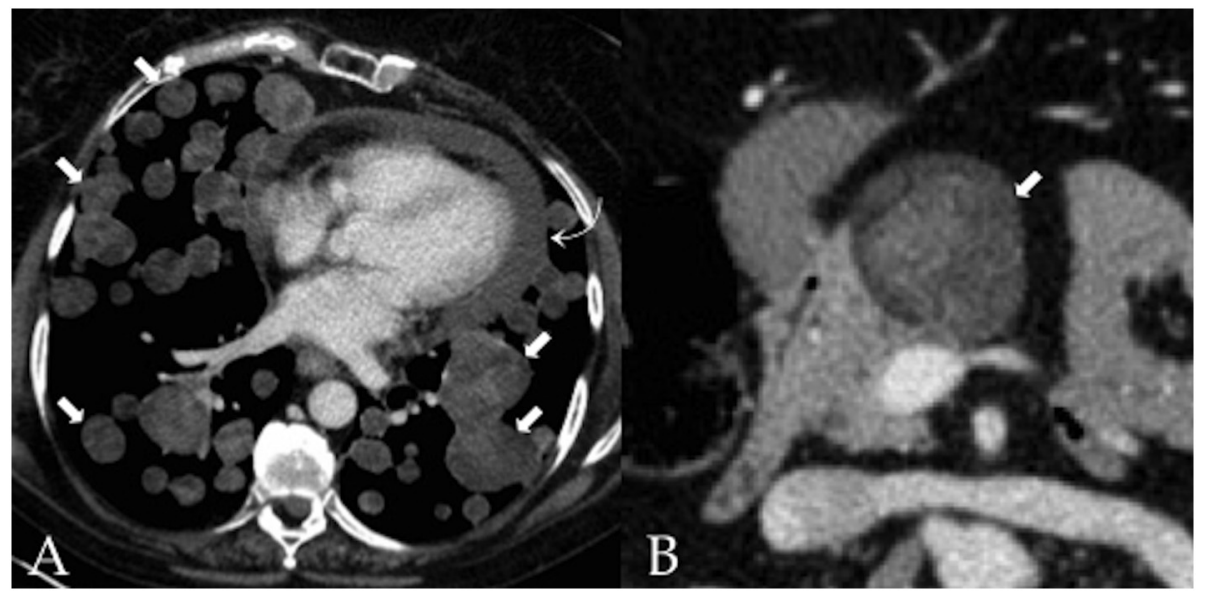

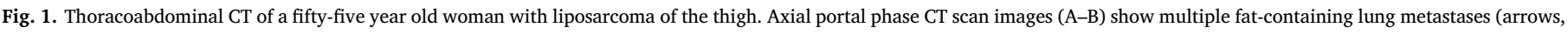
A) and a fat-containing pancreatic metastasis (arrow, B). Note also the pericardial effusion (curved arrow, A).

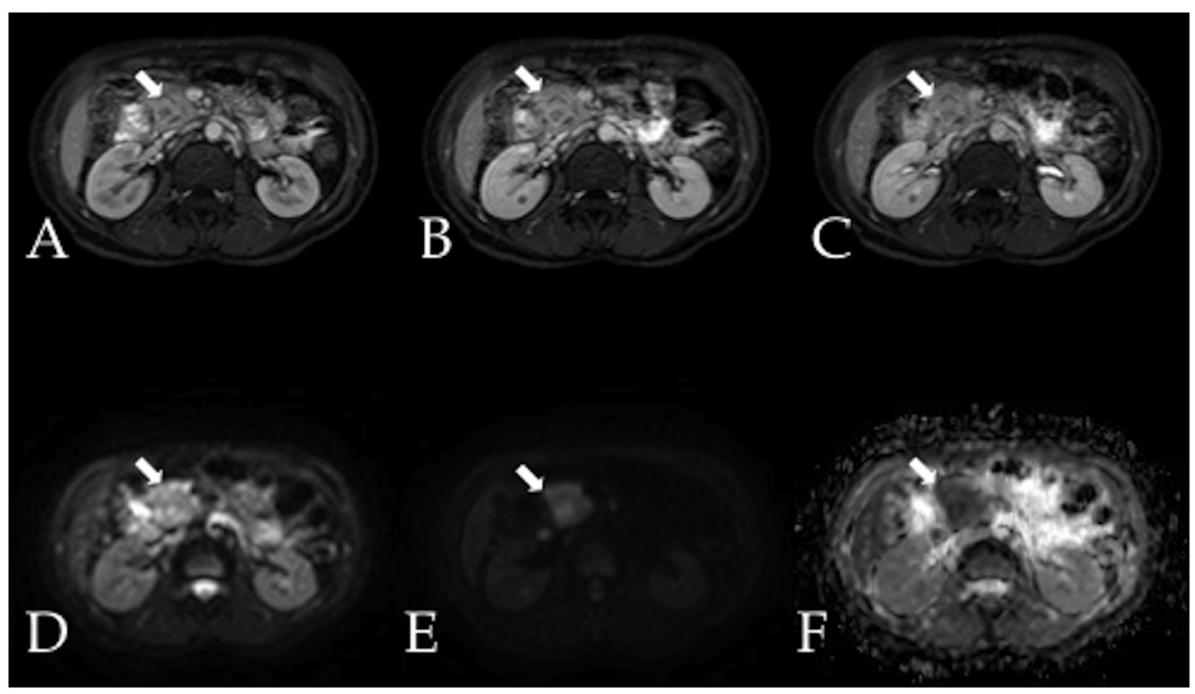

Fig. 2. Abdominal MR scan of a 52-year-old woman with breast carcinoma and isolated pancreatic metastasis. Arterial phase fat-saturated volumetric T1-weighted image (A) shows a small hypovascular metastasis in the head of the pancreas (arrow). Portal (B) and late (C) phase fat-saturated volumetric T1-weighted images show persistent hypointensity of pancreatic metastasis (arrow). The b0 (D) and b600 (E) diffusion-weighted images show slight hyperintensity of the pancreatic metastasis (arrow), which demonstrates restricted pattern of diffusion on ADC map (F).

and PDAC. A $P$-value $<0.05$ was considered statistically significant. Statistical analysis was performed using SPSS 20.0 software.

\section{Results}

A total of 24 patients were positive for PM in our study based on consensus review. We found a total of 54 metastatic lesions to the pancreas. In these patients, the primary malignancy was lung carcinoma in 8/24 (33\%), followed by renal cell carcinoma in 6/24 (25\%). At the time of the detection of PM, 18/24 patients (75\%) had metastases to other organs. The location of primary malignancies and extrapancreatic metastases are shown in Table 1.

Six patients had synchronous PM at the time of the initial diagnosis of the primary tumor. In 18 patients PM was diagnosed during followup imaging, after an average of 18 months from the initial staging evaluation. PM were most commonly multiple (13/24 patients, 54\%), followed by solitary $(9 / 24,38 \%)$ and diffuse $(2 / 24,8 \%)$. In our series, 13 patients with PM and 12 with PDAC underwent MR in addition to CT. In all cases, both PM and PDAC had low signal intensity on T1weighted images, intermediate signal intensity on T2-weighted images, and high signal intensity on high b-value DWI images. The additional features of PM and PDAC are shown in Table 2.

We found a statistically significant difference between PM and PDAC on the basis of location $\left(\chi^{2}=20.3, P<0.001\right)$, margins $\left(\chi^{2}=18.3, P<0.001\right)$, enhancement on arterial phase $\left(\chi^{2}=8.3\right.$,
$P=0.004)$, rim enhancement $\left(\chi^{2}=13.6, P<0.001\right)$, pancreatic duct dilatation $\left(\chi^{2}=6.6, P=0.01\right)$, common bile duct dilatation $\left(\chi^{2}=8.4\right.$, $P=0.003)$, vascular involvement $\left(\chi^{2}=5.1, P=0.02\right)$, parenchymal atrophy $\left(\chi^{2}=11.1, P<0.001\right)$, and peripancreatic fluid $\left(\chi^{2}=4.4\right.$, $P=0.03)$. Conversely, no significant differences were found on the basis of appearance on unenhanced CT, portal phase enhancement, pancreatitis, calcifications, MR appearance on T1 weighted, T2 weighted, and DWI images.

Among PM, 9/24 patients (37\%) had solitary pancreatic lesions, of which in six cases were the only sites of metastatic spread of disease. On arterial phase, they were hypervascular in 4/9 lesions (44\%) compared to the normal enhanced pancreas; in 3/9 lesions (33\%), we found a peripheral rim enhancement on the venous phase. Intratumoral calcifications were found in 1 case $(11 \%)$. Pancreatic duct dilatation was noted in 1 case $(11 \%)$, while common bile duct dilatation, vascular involvement, and parenchymal atrophy were not demonstrated in any case.

Figs. 1-6 show some representative cases from our study population.

\section{Discussion}

Although PM are rare, their diagnosis should be suspected when a pancreatic lesion is detected in a patient with a history of malignancy. In our study, the most frequent primary malignancies that metastasized 


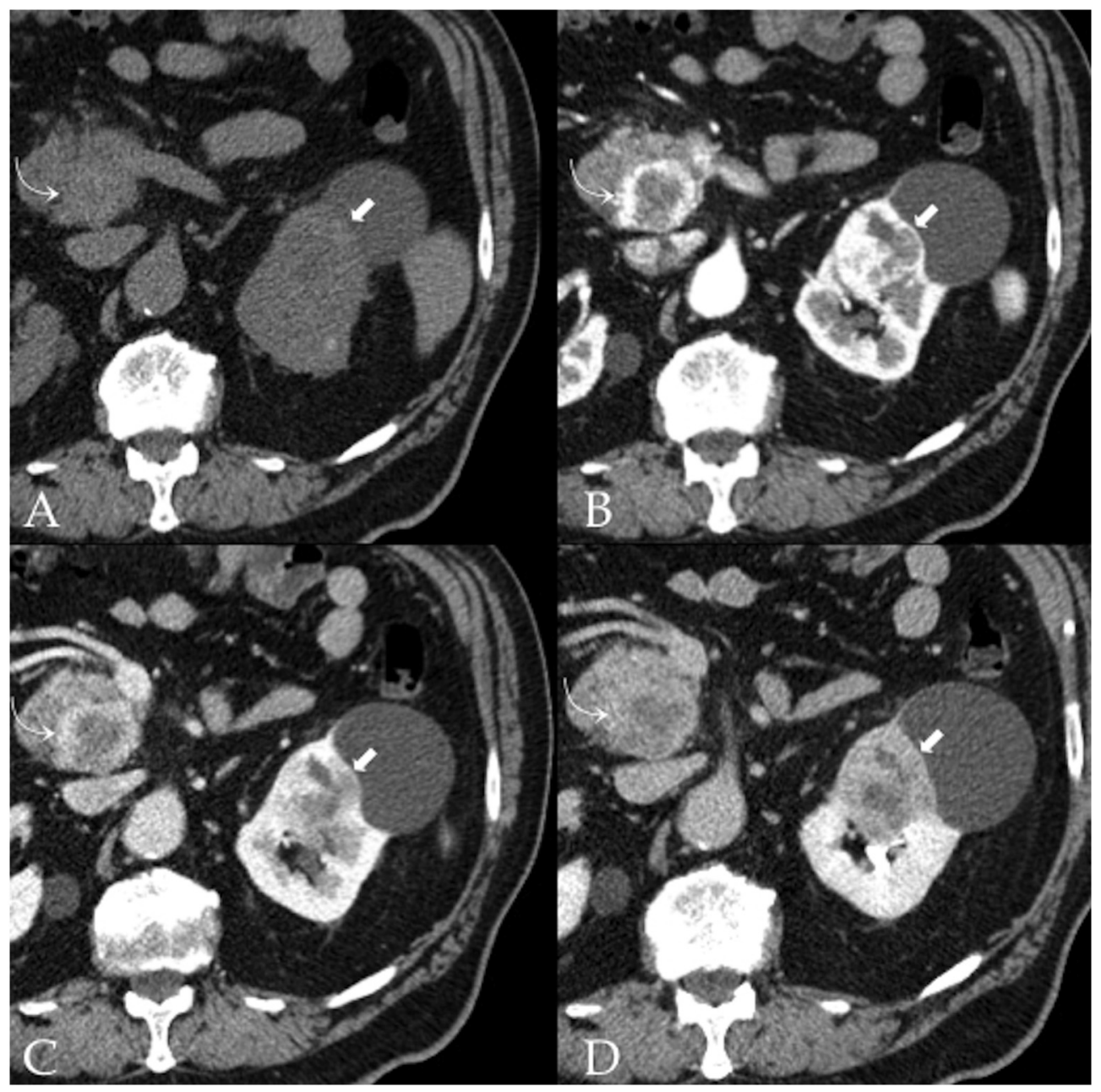

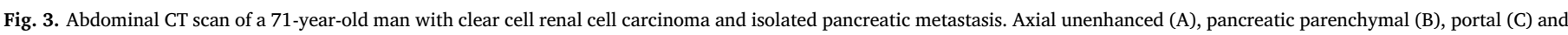

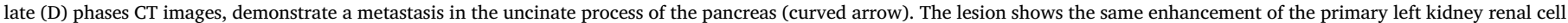
carcinoma (arrow).

to the pancreas were lung cancer and renal cell carcinoma, in line with previous studies [20]. PM may be recognized during the initial imaging staging, at routine follow-up or when imaging is performed for specific signs or symptoms, such as jaundice or abdominal pain [20,21]. PM are commonly associated with disseminated disease and poor prognosis; indeed, a median survival of 8.7 months has been reported in the literature [22]. However, there may be a long latency period between the diagnosis of the primary tumor and the appearance of PM. Minni et al. reported that $77.6 \%$ of patients developed PM metachronously at an average interval of 9.2 years after the diagnosis of the primary tumor [11]. In these cases in particular, CT and MR might help to differentiate a PM from a second primary pancreatic tumor. In our study, PM were more frequently multiple with well-defined margins and showed variable behavior after contrast injection, rare vascular involvement, parenchymal atrophy, and pancreatic or bile ducts dilatation. Moreover, we found a statistically significant difference between PM and PDAC on the basis of location, margins, enhancement on arterial phase, rim enhancement, pancreatic duct dilatation, common bile duct dilatation, vascular involvement, parenchymal atrophy, and peripancreatic fluid.

There are three patterns of PM described in the literature: solitary metastasis, multiple metastases, and diffuse involvement. Although in our study the multiple pattern was slightly more frequent than the single pattern, the latter, characterized by a localized and well-defined mass, was the most common in previous studies [12]. The multiple pattern is characterized by the presence of more than a single lesion and has been previously reported in $5 \%-10 \%$ of cases. The diffuse pattern is characterized by diffuse enlargement of the pancreas with an infiltrative mass; it has been reported in $15 \%-44 \%$ of cases [12-15]. It is well known that PDAC most commonly involves the pancreatic head, as also shown in our series. Conversely, our results showed PM had no predilection for a particular segment of the pancreas, in line with previous studies [23]. On unenhanced CT most PM were hypo- or isoattenuating to normal pancreatic parenchyma, with no significant differences with PDAC. Calcifications and cystic degeneration are other findings which can be observed in PM on unenhanced CT [16]. Similarly, no significant differences between PM and PDAC were observed on unenhanced MR images. PM and PDAC were always hypointense in comparison with the normal high signal of normal gland tissue on unenhanced T1-weighted images, both with and without fat saturation [22]. On T2-weighted and high b-values diffusion-weighted images, the lesions were slightly heterogeneous and moderately hyperintense [12]. Some authors reported that PM from renal cell carcinoma may show intracellular lipid which can be identified through chemical shift MR as a drop of signal on out-of-phase images [24]. Nevertheless, in our series we did not observe this sign in any of our cases. Following intravenous contrast media injection, the behavior of PM may be variable. Smaller lesions $(<1.5 \mathrm{~cm})$ may be hypervascular as compared with the normal parenchyma, while larger lesions $(>1.5 \mathrm{~cm})$ may show peripheral 

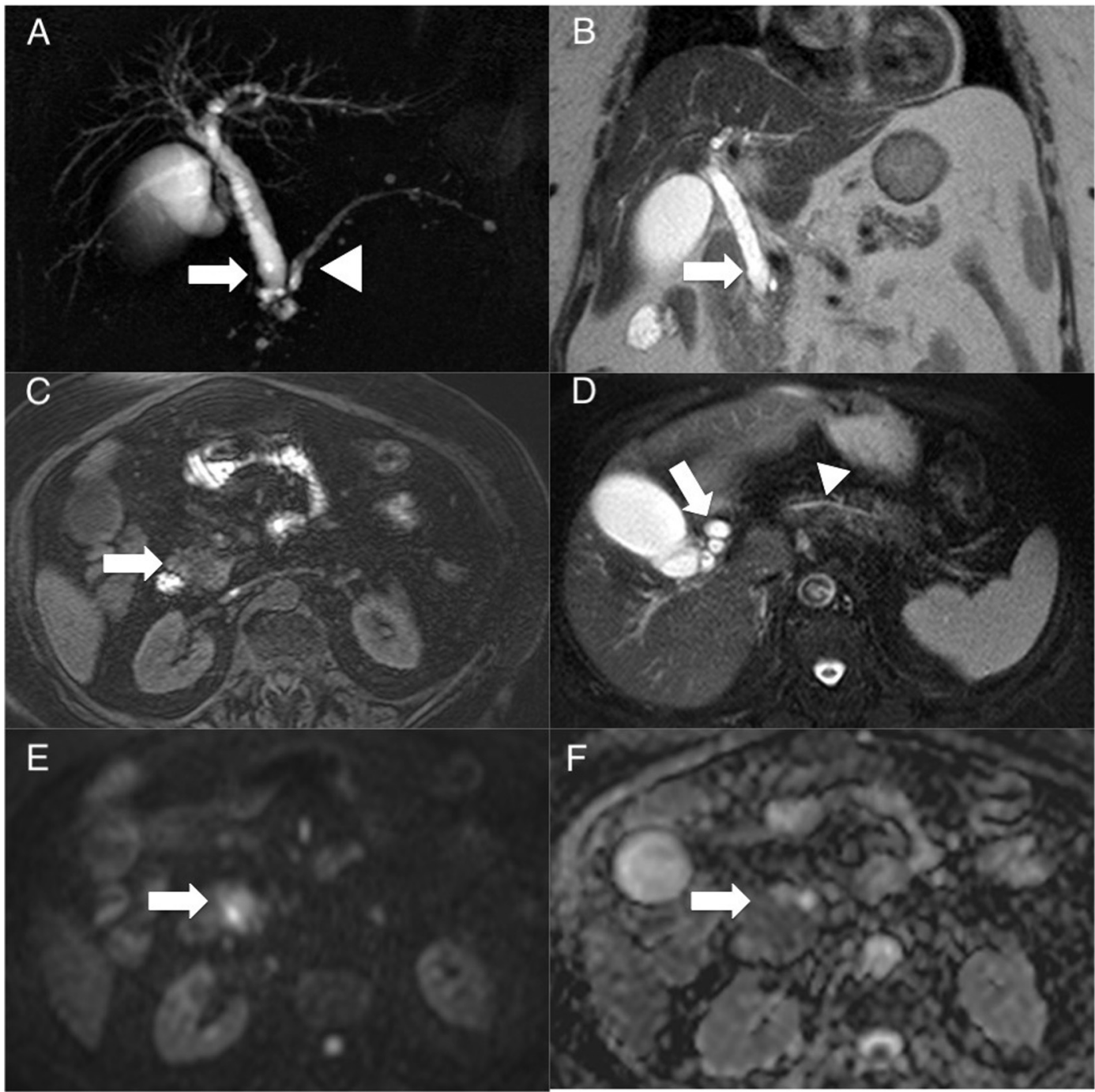

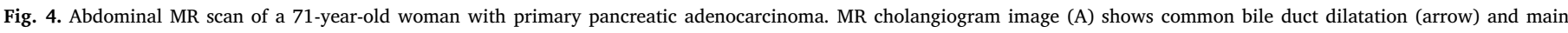

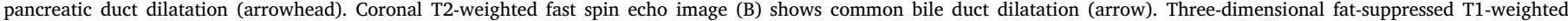

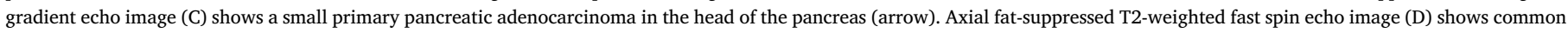

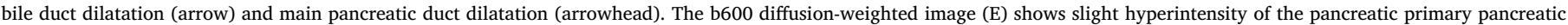
adenocarcinoma (arrow), which demonstrates restricted pattern of diffusion on ADC map (F).

enhancement with central low attenuation/hypointensity mainly due to necrosis; most other lesions are usually hypovascular [25-27]. In our series, most PM were hypovascular and most showed heterogeneous enhancement on the venous phase. Moreover, a frequent feature of PM was rim enhancement, observed in $41 \%$ of cases. Conversely, only one patient (3\%) with PDAC showed rim enhancement after contrast injection. Peripheral enhancement has been previously described as a typical feature of PM, especially from renal cell carcinoma, and can be used to differentiate them from PDAC [28]. As reported by Low et al., this sign is related to the fact that PM parasitize blood supply from surrounding structures, thereby receiving greater perfusion in the periphery of the lesion than in the center [28]. The differential diagnoses of hypervascular PM are primary pancreatic neuroendocrine tumor, intrapancreatic accessory spleen, and vascular lesions such as arteriovenous fistulas or aneurysms of the splenic artery [27,29,30]. Isolated PM from renal cell carcinoma may occur after a long disease free interval $[23,31]$, especially from the clear cell type of primary tumor, and should be differentiated from primary pancreatic endocrine tumor, which may be an incidental finding on cross sectional imaging. Indeed, large non-functional pancreatic neuroendocrine tumors may be asymptomatic for a long time, while small functional neuroendocrine tumors may develop symptoms secondary to hormone secretion [32]. Kanga TW et al. evaluated the usefulness of contrast-enhanced CT with perfusion imaging [33]. They concluded that tumor multiplicity and relative percentage washout value of tumor enhancement with a specific cut-off of $19 \%$ could be helpful for differentiating PM of renal cell carcinoma from hypervascular pancreatic endocrine tumors [33]. The differential diagnoses of hypovascular PM are primary pancreatic carcinoma, lymphomas, and focal pancreatitis. As shown in our and previous studies, it is possible to differentiate PM from PDAC, because PDAC is often associated with other features, including dilatation of the upstream pancreatic duct and/or bile ducts, pancreatic parenchymal atrophy, and mesenteric/splenic vessel involvement [1,19]. Conversely, as demonstrated by our results, these features are less commonly associated with PM. Indeed, in solitary PM pancreatic duct dilatation was rare, while common duct dilatation was not observed. Neither vascular involvement nor parenchymal atrophy was encountered in a case of solitary PM. Furthermore, it is important to emphasize that a history of 


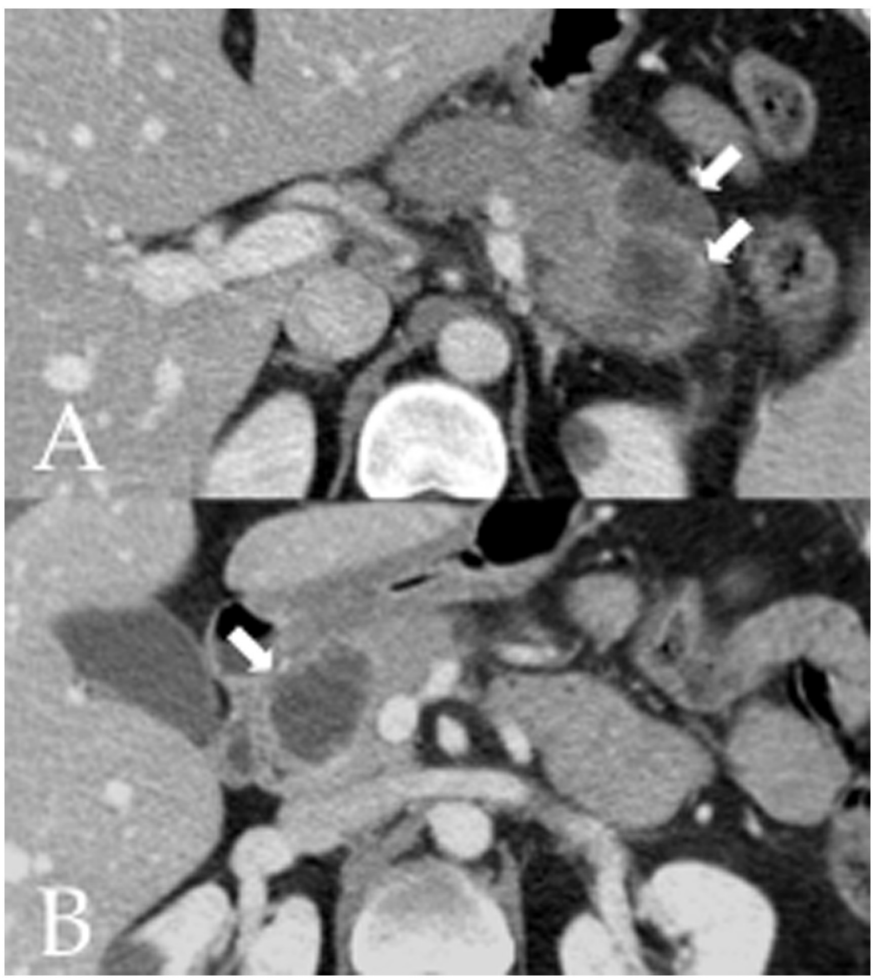

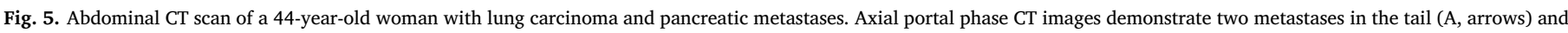

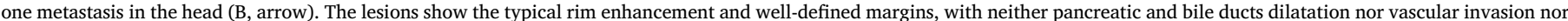
parenchymal atrophy.

malignancy and evaluation of previous imaging are helpful to make a correct diagnosis, with biopsy in controversial cases.

Our study had some limitations. Our study was retrospective and our patient population was relatively small. Secondly, our study had pathologic proof of PM in only six patients. However, these limitations appear inevitable when dealing with a disease as rare as PM.

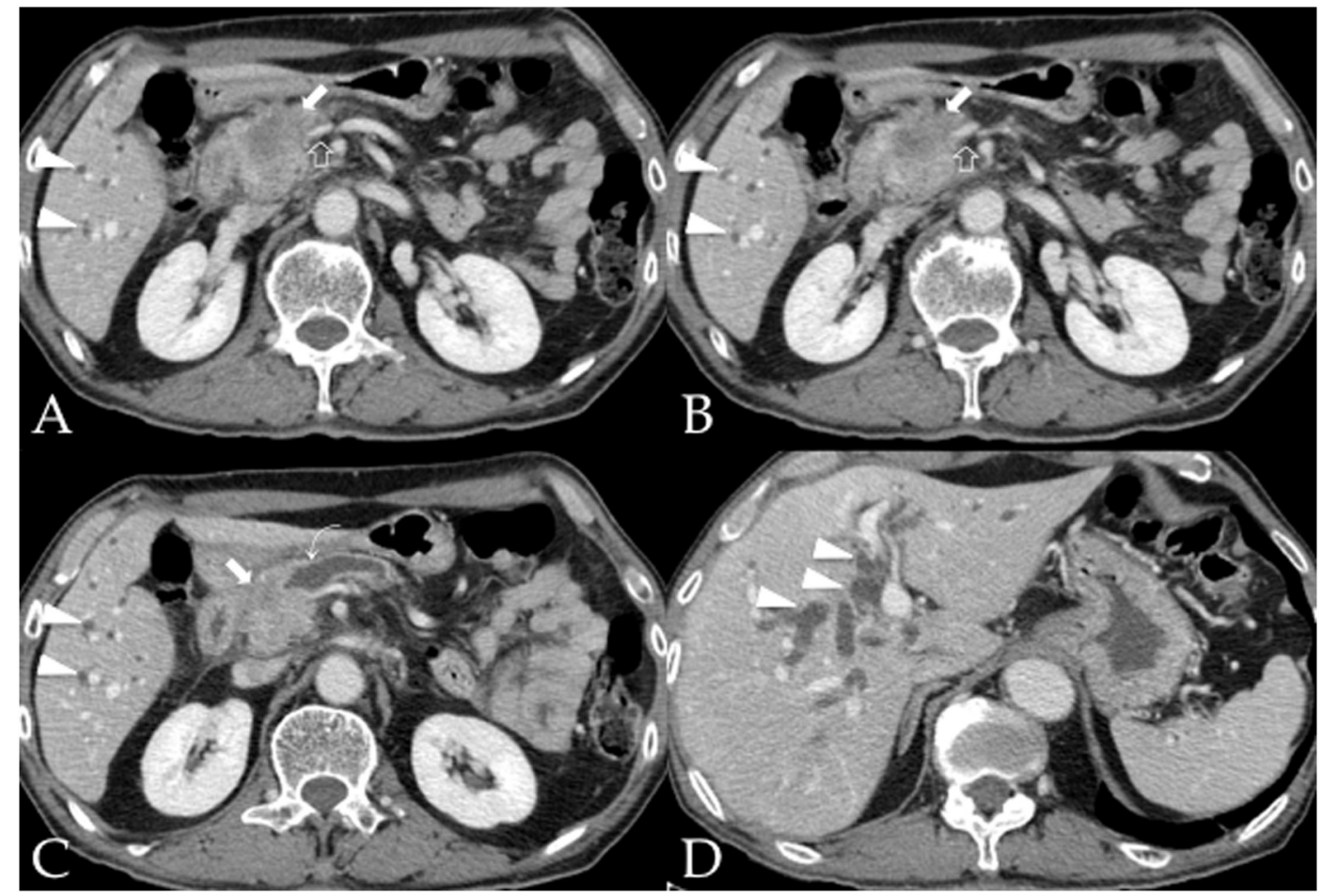

Fig. 6. Abdominal CT scan of a 70-year-old woman with primary pancreatic adenocarcinoma. Axial portal phase CT images demonstrate a hypovascular lesion of the head of the pancreas with ill-defined margins (A-B-C, arrow), associated with main pancreatic duct dilatation (C, curved arrow), bile ducts dilatation (A-B-C-D, arrowheads), and atrophy of the body of the pancreas (C). Note also the involvement of the inferior mesenteric vein, which is narrowed (A-B, hollow arrow). 


\section{Conclusions}

In conclusion, $\mathrm{PM}$ are rare lesions, in our series most commonly seen in patients with lung cancer and renal cell carcinoma. Accurate diagnosis of these lesions may be challenging and biopsy is often required to achieve the correct diagnosis. Imaging features might be helpful to differentiate PM from PDAC. However, the oncological history and evaluation of previous imaging studies are helpful in the diagnosis and management of PM.

\section{Conflicts of interest}

None.

\section{Ethical approval}

All procedures performed in studies involving human participants were in accordance with the ethical standards of the institutional and/ or national research committee and with the 1964 Helsinki declaration and its later amendments or comparable ethical standards. For this type of study formal consent is not required.

\section{References}

[1] Tan CH, Tamm EP, Marcal L, Balachandran A, Charnsangavej C, Vikram R, et al. Imaging features of hematogenous metastases to the pancreas: pictorial essay. Cancer Imaging 2011;11:9-15.

[2] Ballarin R, Spaggiari M, Cautero N, De Ruvo N, Montalti R, Longo C, et al. Pancreatic metastases from renal cell carcinoma: the state of the art. World $J$ Gastroenterol 2011;17:4747-56.

[3] Adsay NV, Andea A, Basturk O, Kilinc N, Nassar H, Cheng JD. Secondary tumors of the pancreas: an analysis of a surgical and autopsy database and review of the literature. Virchows Arch 2004;444:527-35.

[4] Cheng SK, Chuah KL. Metastatic renal cell carcinoma to the pancreas: a review. Arch Pathol Lab Med 2016;140:598-602.

[5] Cwik G, Wallner G, Skoczylas T, Ciechanski A, Zinkiewicz K. Cancer antigens 19-9 and 125 in the differential diagnosis of pancreatic mass lesions. Arch Surg 2006;141:968-73.

[6] Laghi A, Bellini D, Rengo M, Accarpio F, Caruso D, Biacchi D, et al. Diagnostic performance of computed tomography and magnetic resonance imaging for detecting peritoneal metastases: systematic review and meta-analysis. Radiol Med 2017;122:1-15.

[7] Yeh R, Steinman J, Luk L, Kluger MD, Hecht EM. Imaging of pancreatic cancer: what the surgeon wants to know. Clin Imaging 2016;42:203-17.

[8] Galia M, Albano D, Tarella C, Patti C, Sconfienza LM, Mulè A, et al. Whole body magnetic resonance in indolent lymphomas under watchful waiting: the time is now. Eur Radiol 2017. http://dx.doi.org/10.1007/s00330-017-5071-x.

[9] Zaheer A, Wadhwa V, Oh J, Fishman EK. Pearls and pitfalls of imaging metastatic disease from pancreatic adenocarcinoma: a systematic review. Clin Imaging 2015;39:750-8.

[10] Ding Y, Zhou J, Sun H, He D, Zeng M, Rao S. Contrast-enhanced multiphasic CT and
MRI findings of adenosquamous carcinoma of the pancreas. Clin Imaging 2013;37:1054-60.

[11] Minni F, Casadei R, Perenze B, Greco VM, Marrano N, Margiotta A, et al. Pancreatic metastases: observations of three cases and review of the literature. Pancreatology 2004:4:509-20.

[12] Ascenti G, Visalli C, Genitori A, Certo A, Pitrone A, Mazziotti S. Multiple hypervascular pancreatic metastases from renal cell carcinoma: dynamic MR and spiral CT in three cases. Clin Imaging 2004;28:349-52.

[13] Scatarige JC, Horton KM, Sheth S, Fishman EK. Pancreatic parenchymal metastases: observations on helical CT. AJR Am J Roentgenol 2001;176:695-9.

[14] Ferrozzi F, Bova D, Campodonico F, Chiara FD, Passari A, Bassi P. Pancreatic metastases: CT assessment. Eur Radiol 1997;7:241-5.

[15] Muranaka T, Teshima K, Honda H, Nanjo T, Hanada K, Oshiumi Y. Computed tomography and histologic appearance of pancreatic metastases from distant sources. Acta Radiol 1989;30:615-9.

[16] Pan B, Lee Y, Rodriguez T, Lee J, Saif MW. Secondary tumors of the pancreas: a case series. Anticancer Res 2012;32:1449-52.

[17] Ahmed S, Johnson PT, Hruban R, Fishman EK. Metastatic disease to the pancreas: pathologic spectrum and CT patterns. Abdom Imaging 2013;38:144-53.

[18] Triantopoulou C, Kolliakou E, Karoumpalis I, Yarmenitis S, Dervenis C. Metastatic disease to the pancreas: an imaging challenge. Insights Imaging 2012;3:165-72.

[19] Palmowski M, Hacke N, Satzl S, Klauss M, Wente MN, Neukamm M, et al. Metastasis to the pancreas: characterization by morphology and contrast enhancement features on CT and MRI. Pancreatology 2008;8:199-203.

[20] Sweeney AD, Wu MF, Hilsenbeck SG, Brunicardi FC, Fisher WE. Value of pancreatic resection for cancer metastatic to the pancreas. J Surg Res 2009;156:89-198.

[21] Showalter SL, Hager E, Yeo CJ. Metastatic disease to the pancreas and spleen. Semin Oncol 2008;35:160-71.

[22] Roland CF, Van Heerden JA. Nonpancreatic primary tumors with metastasis to the pancreas. Surg Gynecol Obstet 1989;168:345-7.

[23] Sikka A, Adam SZ, Wood C, Hoff F, Harmath CB, Miller FH. Magnetic resonance imaging of pancreatic metastases from renal cell carcinoma. Clin Imaging 2015;39:945-53.

[24] Carucci LR, Siegelman ES, Feldman MD. Pancreatic metastasis from clear cell renal carcinoma: diagnosis with chemical shift MRI. J Comput Assist Tomogr 1999;23(6):934.

[25] Crippa S, Angelini C, Mussi C, Bonardi C, Romano F, Sartori P, et al. Surgical treatment of metastatic tumors to the pancreas: a single center experience and review of the literature. World J Surg 2006;30:1536-42.

[26] Sperti C, Pasquali C, Liessi G, Pinciroli L, Decet G, Pedrazzoli S. Pancreatic resection for metastatic tumors to the pancreas. J Surg Oncol 2003;83:161-6.

[27] Law CH, Wei AC, Hanna SS, Al-Zahrani M, Taylor BR, Greig PD, et al. Pancreatic resection for metastatic renal cell carcinoma: presentation, treatment, and outcome. Ann Surg Oncol 2003;10:922-6.

[28] Low G, Panu A, Millo N, Leen E. Multimodality imaging of neoplastic and nonneoplastic solid lesions of the pancreas. Radiographics 2011;31:993-1015.

[29] Mechó S, Quiroga S, Cuéllar H, Sebastià C. Pancreatic metastasis of renal cell carcinoma: multidetector CT findings. Abdom Imaging 2009;34:385-9.

[30] Ninan S, Jain PK, Paul A, Menon KV. Synchronous pancreatic metastases from asymptomatic renal cell carcinoma. JOP 2005;6:26-8.

[31] Galia M, Albano D, Bruno A, Agrusa A, Romano G, Di Buono G, et al. Imaging features of solid renal masses. Br J Radiol 2017;90:20170077.

[32] McKenna LR, Edil BH. Update on pancreatic neuroendocrine tumors. Gland Surg 2014;3:258-75.

[33] Kang TW, Kim SH, Lee J, Kim AY, Jang KM, Choi D, et al. Differentiation between pancreatic metastases from renal cell carcinoma and hypervascular neuroendocrine tumour: use of relative percentage washout value and its clinical implication. Eur J Radiol 2015;84:2089-96. 